\title{
The study on the medium parton distribution from momentum kick model in Heavy-lon Collisions
}

\author{
Soyeon Cho ${ }^{a, *}$ and Jin-Hee Yoon ${ }^{a}$ \\ ${ }^{a}$ Department of physics, Inha University, \\ 100 Inha-ro, Michuhol-gu, Incheon 22212, Republic of Korea
}

E-mail: jinyoon@inha.ac.kr

The ridge-like structure found in two-particle correlation from proton-proton collisions is one of the hot topics in high-energy heavy-ion physics. Because the scale of pp collisions is not large enough to generate a high-temperature and high-density medium called QGP, this phenomenon cannot be suitably understood through hydrodynamics, unlike in nucleus-nucleus collision. In a meanwhile, jet particles lose considerable energy while moving through the collisions with partons in the medium. The momentum transferred from jets to medium partons is in the direction of jets' motion, which might produce the collective motion of the medium, such as the ridge. In this sense, the momentum kick model has been tested in the nucleus-nucleus and pp collisions at various energies. [1]

For its validity we try to apply this model to the correlation observable and we need the initial parton distribution of the medium. We have adopted several distribution functions: MaxwellBoltzmann(MB), Juttner-Synge(JS), and phenomenological parton distribution functions from the soft scattering model (phPDs) [2][3] and from the hard scattering model (phPDh) [4][5]. However, the MB and JS can not explain the parton momentum distribution over a wide range of pseudo-rapidity and phPDs does not describe the lightcone variable distribution. Therefore we proceed to use phPDh relatively in detail.

In this study, we find the optimal values of model parameters by fitting to the simulated data for pp collisions at $\sqrt{s_{N N}}=2.76 \mathrm{TeV}$ from PYTHIA8. We compare not only the the transverse momentum and rapidity distribution but also the lightcone variable distribution. Using these settings, we calculate the two-particle correlations and compare them to the experimental results.

The Ninth Annual Conference on Large Hadron Collider Physics - LHCP2021

7-12 June 2021

Online

${ }^{*}$ Speaker 


\section{Introduction}

The Ridge structure represents the pseudo-rapidity $(\eta)$-independent shape in two-particle angular correlations at High-energy Heavy-ion collision experiments, for a large $\eta$ range. This structure in AA collisions is an evidence for Quark-Gluon Plasma (QGP), but it is recently reported even in high-multiplicity pp collisions. Small systems like pp collisions, even with high multiplicity, are not expected to produce a thermalized and condensed medium, called QGP, unlike AA collisions. Thus, we try to understand the Ridge structure in high-multiplicity small systems via kinematic interaction between jets and medium partons. Jets lose their energy and momentum as they pass through the medium. Those energies and momenta lost by highly energetic jets are transferred to medium partons and tend to align them along jet-direction, resulting in a collective motion like the Ridge structure. To describe this kinematic process, we need an information on parton's momentum distribution of the medium created from the collision and we test several distribution functions.

\section{Characteristics of PDF}

In the previous study [1], two symmetric double scattering processes between jets and medium generate a constructive interference, resulting in a collective motion of medium partons along jet-direction. Based on this result, we expect that Feynman amplitudes from the two symmetric diagrams in Figure 1 similarly behave bringing out a collective motion of medium. As an initial distribution of medium partons, we try various functions - Maxwell-Boltzmann(MB), Juttner-Synge(JS), phenomenological parton distribution functions from the soft scattering model (phPDs) [2][3], and that from the

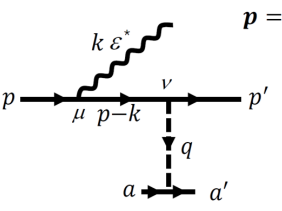

(a)

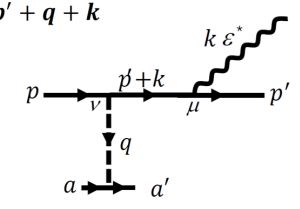

(b)

Figure 1: Feynman diagrams for two symmetric Bremsstrahlung processes - photons are emitted (a) before collision and (b) after collision. hard scattering model (phPDh) [4][5] - to fit the PYTHIA8 monash simulation in three aspects; rapidity $\left(y_{a}\right)$ distribution, transverse momentum $\left(a_{T}\right)$ distribution, and lightcone variable $(x)$ distribution. In Figure 2, panel (a) shows the $\eta$-distributions of all four models and the MB and the JS are not suitable to describe the whole range of $\eta$. Panel (b) displays the $a_{T}$-distributions and the JS behaves differently compared to the simulation data. Panel (c), shows the $x$-distribution and the phPDs fails for high $x$. Therefore, the phPDh is the only choice available and we make the calculations using the phPDh. It is given by

$$
f\left(y_{a}, a_{T}\right)=A(1-x)^{a}\left[1-(1-q) \frac{m_{T}}{T}\right]^{\frac{1}{1-q}},
$$

where $A$ is a normalization constant and $m_{T}$ is the transverse mass of a parton in medium. $a, q$, $T$ are free parameters which characterize the phPDh: $a$ is the fallout parameter which decides the shape of rapidity distribution, $q$ is the non-extensive parameter phenomenologically equivalent to the quasi power law, and $T$ is the temperature of the system. As you can see from the Figure 2 (d)-(f), $q$ influences $y_{a^{-}}$and $a_{T}$-distributions significantly and $a$ affects the $y_{a^{-}}$and $x$-distributions mildly. $T$-dependence is not shown but it affects $a_{T}$ - and $y_{a}$-distributions weakly. Overall, $\eta$-distribution 
(a)

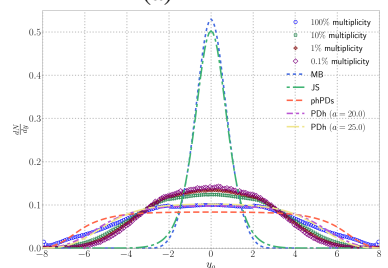

(d)

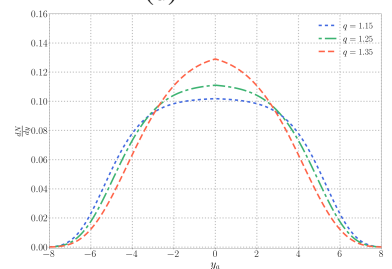

(b)

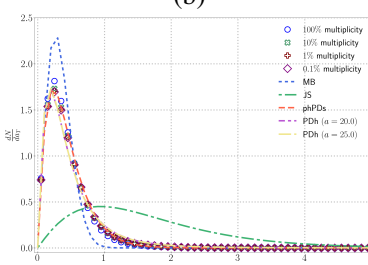

(e)

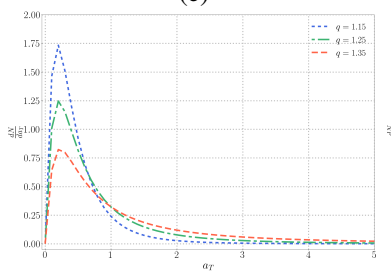

(c)

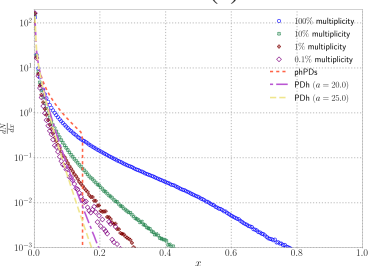

(f)

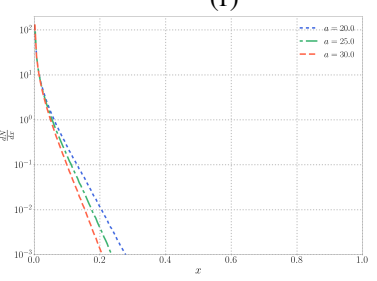

Figure 2: Various distribution functions on (a) rapidity,(b) transverse momentum, and (c) lightcone variable, compared with the PYTHIA8 monash simulation. Bottom panels((d)-(f)) shows the major-dependencies of the phPDh on free parameters, respectively.

(a)

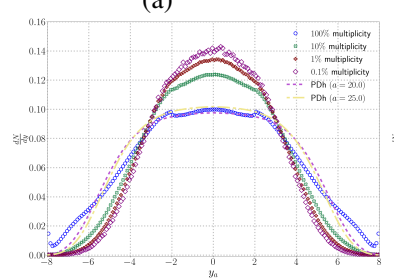

(b)

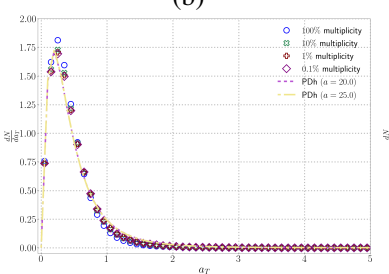

(c)

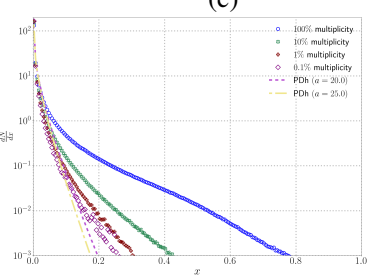

Figure 3: phPDh distribution functions with selected optimal values of $a=20, q=1.15$, and $T=0.15 \mathrm{GeV}$, compared with the simulation data.

is adjusted by $q$, $x$-distribution by $a$, and $a_{T}$-distribution mostly by $q$ but tuned finely by $T$. Finally, we find the optimal values of $a=20 \sim 25, q=1.15$, and $T=0.15 \mathrm{GeV}$ and the phPDh distribution functions are compared with the PYTHIA8 monash simulations in the Figure 3. The phPDh describes well the $y_{a}$-distribution for $100 \%$ multiplicity, the $a_{T}$-distribution for all multiplicity, and the $x$-distribution for $1 \% \sim 0.1 \%$ multiplicity, but does not reproduce $x$-distribution for $100 \%$ multiplicity. Further study on this dependence check is necessary.

\section{Results \& Conclusion}

We calculate the correlation using the phPDh with these optimized values. We formulate a two-particle correlation function as following:

$$
C\left(\mathbf{a}_{1}, \mathbf{a}_{2}\right)=\frac{P_{f}\left(\mathbf{a}_{1}, \mathbf{a}_{2}\right)}{P_{i}\left(\mathbf{a}_{1}\right) \cdot P_{i}\left(\mathbf{a}_{2}\right)}=\frac{P_{f}\left(\mathbf{a}_{1}\right) \cdot P_{f}\left(\mathbf{a}_{2}\right)}{P_{i}\left(\mathbf{a}_{1}\right) \cdot P_{i}\left(\mathbf{a}_{2}\right)} .
$$

In Eq. $2, P_{i}(\mathbf{a})$ is the probability of medium partons before interaction with jet particle and $P_{f}(\mathbf{a})$ is that after interaction. We compare the calculations to the experimental results in Figure 4. 

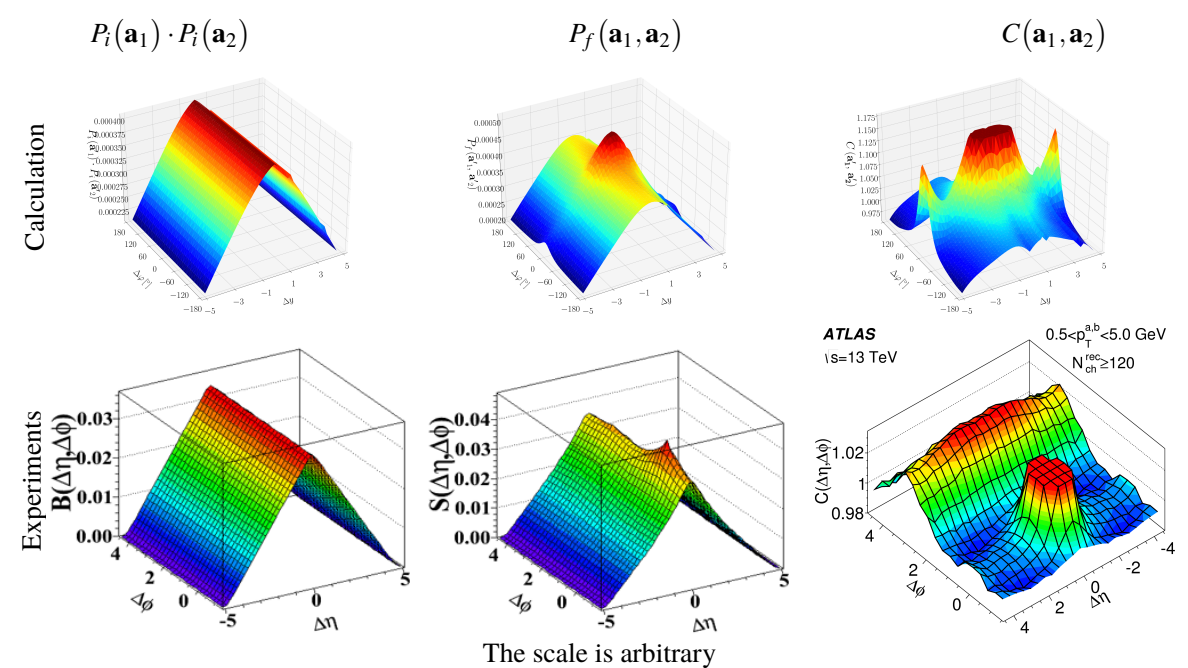

Figure 4: Comparison of the phPDh calculations(upper panels) and the experimental results(lower panels); Left column shows two-particle pair probability of medium partons before interaction with jet particle \& background distribution [6], middle column shows two-particle pair probability of final medium partons after interaction with jet particle \& signal distribution [6], and right column shows correlation [7].

$P_{i}\left(\mathbf{a}_{1}\right) \cdot P_{i}\left(\mathbf{a}_{2}\right)$ and $P_{f}\left(\mathbf{a}_{1}, \mathbf{a}_{2}\right)$ correspond to $B(\Delta \eta, \Delta \phi)$ and $S(\Delta \eta, \Delta \phi)$, respectively. They behave similarly but the correlations look little different. One thing to note is that our model results from a single scattering process and it reproduces only the near-side ridge. It also has a rapidly uprising behavior around the boundaries in $\Delta y$ which might be resulted from the edge effect in numerical calculations, which needs to be examined further. Overall we can see a ridge-like behavior for $\Delta y>|3|$.

\section{Summary \& Outlooks}

In this study, we test various distribution functions to describe the initial medium parton distribution. We choose phenomenological parton distribution functions from the hard scattering model (phPDh) and check its characteristics. We find optimal values of parameters in phPDh by comparing the phPDh model calculations to the PYTHIA simulation data. Also, we calculate the correlation using the phPDh and compare it to experimental results. Our results can describe background and signal distributions in experiments well but not the correlation, especially rapid uprising beyond $|y|=5$. We will look into this in more detail and compare it with other simulations such as PYTHIA with string shoving, EPOS, etc. Also, we plan to include jet components in our calculation.

\section{Acknowledgement}

This work was supported by the National Research Foundation of Korea(NRF) grant funded by the Korea government(MSIT)(No. NRF-2008-00458) and the Inha University Research Fund. Authors also thank the KISTI (GSDC) for computing resources supplied. 


\section{References}

[1] C.-Y. Wong, Bose-einstein interference in the passage of a jet in a dense medium, Physical Review C 85 (2012) 064909.

[2] C.-Y. Wong, Ridge structure in the $\delta \phi-\delta \eta$ correlation function associated with a near-side jet, Physical Review C 76 (2007) 054908.

[3] C.-Y. Wong, Momentum kick model description of the near-side ridge and jet quenching, Physical Review C 78 (2008) 064905.

[4] C.-Y. Wong and G. Wilk, Tsallis fits to $p_{t}$ spectra and multiple hard scattering in $\mathrm{p} p$ collisions at the lhc, Physical Review D 87 (2013) 114007.

[5] C.-Y. Wong, G. Wilk, L.J. Cirto and C. Tsallis, From qcd-based hard-scattering to nonextensive statistical mechanical descriptions of transverse momentum spectra in high-energy $\mathrm{p} p$ and $\mathrm{p}$ p collisions, Physical Review D 91 (2015) 114027.

[6] V. Khachatryan, A. Sirunyan, A. Tumasyan, W. Adam, T. Bergauer, M. Dragicevic et al., Observation of long-range, near-side angular correlations in proton-proton collisions at the

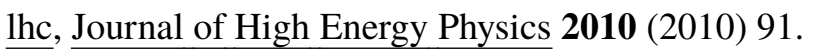

[7] G. Aad, B. Abbott, J. Abdallah, R. Aben, M. Abolins, O. AbouZeid et al., Observation of long-range elliptic azimuthal anisotropies in $\sqrt{s}=13$ and 2.76 tev p p collisions with the atlas detector, Physical review letters 116 (2016) 172301. 